\title{
SEMILINEAR VOLTERRA INTEGRODIFFERENTIAL EQUATIONS WITH NONLOCAL INITIAL CONDITIONS
}

\author{
SERGIU AIZICOVICI AND MARK MCKIBBEN
}

Received 20 June 1999

We establish the global existence of mild solutions to a class of nonlocal Cauchy problems associated with semilinear Volterra integrodifferential equations in a Banach space.

\section{Introduction}

We investigate the global existence of solutions to integrodifferential equations with nonlocal conditions of the general form

$$
\begin{gathered}
u^{\prime}(t)+\int_{0}^{t} a(t-s) A u(s) d s=F(u)(t), \quad 0<t<T, \\
u(0)=g(u)
\end{gathered}
$$

in a Banach space $X$. Here, $A$ is a closed, densely-defined linear unbounded operator on $X, g: C([0, T] ; X) \rightarrow X, a \in L^{1}(0, T)$, and $F: C([0, T] ; X) \rightarrow L^{p}(0, T ; X)$ $(p \in[1, \infty])$ is a continuous hereditary mapping. The unknown $u$ takes values in $X$. As a special case of (1.1), we consider the problem

$$
\begin{gathered}
u^{\prime}(t)+\int_{0}^{t} a(t-s)[A u(s)+f(s, u(s))] d s=h(t), \quad 0<t<T, \\
u(0)=g(u),
\end{gathered}
$$

where $h \in L^{1}(0, T ; X)$ and $f:[0, T] \times X \rightarrow X$. This is obtained if one takes $F(u)(t)=$ $h(t)-\int_{0}^{t} a(t-s) f(s, u(s)) d s$ in (1.1).

Such problems are important from the viewpoint of applications since they cover nonlocal generalizations of integrodifferential equations arising in the mathematical modeling of heat conduction in materials with memory. Byszewski $[6,7]$ initiated the work concerning abstract nonlocal semilinear initial-value problems. He used fixedpoint methods to prove the existence and uniqueness of mild solutions to the Cauchy

Copyright (C) 1999 Hindawi Publishing Corporation Abstract and Applied Analysis 4:2 (1999) 127-139 1991 Mathematics Subject Classification: 45D05, 45K05, 34K30 URL: http://aaa.hindawi.com/volume-4/S1085337599000123.html 
problem

$$
\begin{gathered}
u^{\prime}(t)+A u(t)=f(t, u(t)), \quad 0<t<T, \\
u(0)+g\left(t_{1}, \ldots, t_{p}, u\left(t_{1}\right), \ldots, u\left(t_{p}\right)\right)=u_{0},
\end{gathered}
$$

where $p \in \mathbb{N}, 0<t_{1}<\cdots<t_{p} \leq T$ are given, $u_{0} \in X,-A$ generates a linear $C_{0^{-}}$ semigroup on $X$, while $f:[0, T] \times X \rightarrow X$ and $g:[0, T]^{p} \times X^{p} \rightarrow X$ satisfy Lipschitz conditions. See also $[4,5,8,9,13]$ for related results, including applications to integrodifferential equations. Next, Ntouyas and Tsamatos $[17,18]$ studied nonlocal semilinear problems in the absence of Lipschitz conditions by using compactness arguments. The extension of [6, 7] to the nonlinear case was carried out by Aizicovici and Gao [1]. Recently, Aizicovici and McKibben [3] have extended the results in [17, 18] to the fully nonlinear case under the crucial assumption that $-A$ generates a compact nonlinear semigroup on $X$.

The paper most closely related to the present one is by Lin and Liu [15]. They developed an existence theory for the nonlocal integrodifferential equation

$$
\begin{gathered}
u^{\prime}(t)+A\left[u(t)+\int_{0}^{t} a(t-s) u(s) d s\right]=f(t, u(t)), \quad 0<t<T, \\
u(0)+g\left(t_{1}, \ldots, t_{p}, u\left(t_{1}\right), \ldots, u\left(t_{p}\right)\right)=u_{0}
\end{gathered}
$$

in $X$. Here $A, g, f$, and $u_{0}$ are as in (1.3), and $a(t), 0<t<T$, is a bounded linear operator on $X$.

Our results concerning (1.1) and (1.2) can be viewed as a counterpart to the results in [15], in the sense that the operator $A$ appears now only behind the integral sign. We first establish the existence and uniqueness of mild solutions to (1.1) under Lipschitz conditions on $F$ and $g$. We then replace the Lipschitz conditions by weaker sublinear growth conditions, at the expense of some compactness restrictions.

The outline of the paper is as follows. In Section 2, we review some basic facts concerning resolvent operators and mild solutions for abstract linear integrodifferential equations. Section 3 is concerned with the existence and uniqueness of solutions to (1.1) under Lipschitz conditions on $F$ and $g$, while Section 4 is devoted to the existence theory for (1.1) and (1.2) under compactness assumptions. Finally, two examples involving integro-partial differential equations are discussed in Section 5.

\section{Preliminaries}

For further background and details of this section, we refer the reader to $[10,11,12$, $19,20]$ and the references therein. Let $X$ be a Banach space with norm $\|\cdot\|$, and $A: D(A) \subset X \rightarrow X$ be a linear, closed operator with dense domain $D(A)$. Consider the Volterra integrodifferential equation

$$
\begin{gathered}
u^{\prime}(t)+\int_{0}^{t} a(t-s) A u(s) d s=h(t), \quad 0 \leq t \leq T, \\
u(0)=u_{0} \in D(A),
\end{gathered}
$$


where $a \in L^{1}(0, T)$ and $h \in L^{1}(0, T ; X)$. It is easily seen that (2.1) can be rewritten in the equivalent form

$$
u(t)+b * A u(t)=H(t), \quad 0 \leq t \leq T,
$$

where $b(t)=\int_{0}^{t} a(s) d s, H(t)=u_{0}+\int_{0}^{t} h(s) d s$, and “*” denotes the convolution over $(0, t)$.

Definition 2.1. A function $u \in C([0, T] ; X)$ is called a mild solution of (2.1) if $b * u \in$ $C([0, T] ; Y)$ and $u(t)=H(t)-A(b * u)(t)$, for all $t \in[0, T]$, where $Y$ stands for $D(A)$ equipped with the graph norm.

Definition 2.2. A family $\{S(t)\}_{0 \leq t \leq T}$ of bounded linear operators in $X$ is said to be a resolvent for (2.1) (equivalently (2.2)) if the following conditions are satisfied:

(i) $S(t)$ is strongly continuous on $[0, T]$ and $S(0)=I$;

(ii) $S(t) D(A) \subseteq D(A)$ and $A S(t) x=S(t) A x$, for every $x \in D(A), 0 \leq t \leq T$;

(iii) $S(t) x=x-\int_{0}^{t} b(t-s) A S(s) x d s$, for all $x \in D(A), 0 \leq t \leq T$.

Note that $\|S(t)\|_{\mathscr{B}(X)} \leq M_{S}$ on $[0, T]$ due to (i) and the uniform boundedness principle. (Here $\mathscr{B}(X)$ designates the space of all bounded linear operators on $X$.)

General conditions on $a$ and $A$ that guarantee the existence and uniqueness of a resolvent family for (2.1) can be found in [12].

It is convenient to represent a mild solution of (2.1) using a variation of parameters type formula involving the resolvent $S(t)$. Specifically, we have the following proposition.

Proposition 2.3. Suppose that (2.1) admits a resolvent $S(t)$. Then, for any $x_{0} \in X$ and $h \in L^{1}(0, T ; X)$, equation (2.1) has a unique mild solution $u$, given by

$$
u(t)=S(t) u_{0}+\int_{0}^{t} S(t-s) h(s) d s, \quad \forall t \in[0, T] .
$$

Next we consider (2.1) under the following stronger restrictions on $a$ and $A$ :

(C1) $a \in L^{1}(0, T)$ is positive, nonincreasing, and convex;

(C2) $A$ is a linear, densely-defined, closed, invertible operator such that $A^{-1}$ is compact on $X$;

(C3) either

(i) $-a^{\prime}$ is convex, $X$ is a separable Hilbert space, and $A$ is self-adjoint and strictly positive definite, or

(ii) $a$ is log-convex (with $a^{\prime}$ locally absolutely continuous on $(0, T]$ in the case when $\left.a\left(0^{+}\right)<\infty\right)$, and $-A$ generates a strongly continuous cosine family on $X$.

These conditions ensure (cf. $[10,11,14,19])$ that $(2.1)$ has a resolvent $S(t)$, in the sense of Definition 2.2. Now let $\Phi: L^{1}(0, T ; X) \rightarrow C([0, T] ; X)$ be defined by

$$
\Phi(v)=S * v, \quad \forall v \in L^{1}(0, T ; X) .
$$

The following two compactness results have been established in [2]. 
Proposition 2.4. Let conditions (C1), (C2), and (C3)(either (i) or (ii)) be satisfied. In addition, assume that

$$
a^{\prime}\left(0^{+}\right)=-\infty
$$

Then $\Phi$ maps each uniformly integrable subset of $L^{1}(0, T ; X)$ into a precompact subset of $C([0, T] ; X)$. In particular, $\Phi$ is compact as a map of $L^{p}(0, T ; X)(1<p \leq \infty)$ into $C([0, T] ; X)$.

Proposition 2.5. Let conditions (C1), (C2), and (C3)(either (i) or (ii)) be satisfied. In addition, assume

$$
a\left(0^{+}\right)<\infty
$$

Then the mapping $v \mapsto \Phi(a * v)$ from $L^{1}(0, T ; X)$ into $C([0, T] ; X)$ is compact.

Finally, we recall a useful alternative of the Leray-Schauder principle, which will play a key role in Section 4.

Theorem 2.6 (Schaefer's fixed point theorem [21]). Let $\mathscr{F}: X \rightarrow X$ be a continuous, compact map, and let $\xi(\mathscr{F})=\{x \in X: \lambda x=\mathscr{F} x$, for some $\lambda \geq 1\}$. If $\xi(\mathscr{F})$ is bounded, then $\mathscr{F}$ has a fixed point.

\section{The case of a Lipschitz continuous nonlinearity}

Consider (1.1) in a Banach space $X$ under the following assumptions:

(H1) $A: D(A) \subseteq X \rightarrow X$ is a closed, linear, densely-defined, unbounded operator;

(H2) $a \in L^{1}(0, T)$;

(H3) equation (2.1) admits a resolvent $S(t)$, with $\max _{t \in[0, T]}\|S(t)\|_{\mathscr{B}(X)}=M_{S}$;

(H4) $F: C([0, T] ; X) \rightarrow L^{p}(0, T ; X), 1 \leq p \leq \infty$, is a hereditary map; that is, for each fixed $t \in[0, T]$ and $u \in C([0, T] ; X)$, the value $F(u)(t)$ is allowed to depend only on the restriction of $u$ on $[0, t]$. In addition, there exists $M_{F}>0$ such that

$$
\|F(u)-F(v)\|_{L^{p}(0, T ; X)} \leq M_{F}\|u-v\|_{C([0, T] ; X)}, \quad \forall u, v \in C([0, T] ; X) ;
$$

(H5) $g: C([0, T] ; X) \rightarrow X$ satisfies the following condition: there exists $M_{g}>0$ such that $\|g(u)-g(v)\| \leq M_{g}\|u-v\|_{C([0, T] ; X)}$, for all $u, v \in C([0, T] ; X)$;

(H6) $M_{S}\left(M_{g}+M_{F} T^{1 / q}\right)<1$, where $q$ is the conjugate of $p$ (i.e., $p^{-1}+q^{-1}=1$ ).

Remark 3.1. Naturally, by a mild solution $u \in C([0, T] ; X)$ to (1.1), we mean a mild solution to (2.1) with $u_{0}=g(u)$ and $h=F(u)$. According to Proposition 2.3, $u$ is a mild solution of (1.1) if and only if

$$
u(t)=S(t) g(u)+\int_{0}^{t} S(t-s) F(u)(s) d s, \quad \forall t \in[0, T]
$$


Theorem 3.2. Assume that (H1)-(H6) are satisfied. Then (1.1) has a unique mild solution.

Proof. Let $v \in C([0, T] ; X)$ and consider the problem

$$
\begin{gathered}
u^{\prime}(t)+\int_{0}^{t} a(t-s) A u(s) d s=F(v)(t), \quad 0<t<T, \\
u(0)=g(v) .
\end{gathered}
$$

Define the map $\mathscr{F}: C([0, T] ; X) \rightarrow C([0, T] ; X)$ by $\mathscr{F} v=u_{v}$, where $u_{v}$ is the mild solution to (3.3). We show that $\mathscr{F}$ is a strict contraction. To this end, let $v, w \in C([0, T] ; X)$. By using (2.3), (H2), (H3), (H4), and (H5), together with Hölder's inequality, we obtain

$$
\begin{aligned}
\left\|u_{v}-u_{w}\right\|_{C([0, T] ; X)} & \leq M_{S} M_{g}\|v-w\|_{C([0, T] ; X)}+M_{S} \int_{0}^{t}\|(F v)(s)-(F w)(s)\| d s \\
& \leq M_{S} M_{g}\|v-w\|_{C([0, T] ; X)}+M_{S} T^{1 / q}\|F v-F w\|_{L^{p}(0, T ; X)} \\
& \leq M_{S}\left(M_{g}+M_{F} T^{1 / q}\right)\|v-w\|_{C([0, T] ; X) .}
\end{aligned}
$$

Since $M_{S}\left(M_{g}+M_{F} T^{1 / q}\right)<1$ (cf. (H6)), we conclude that $\mathscr{F}$ is indeed a strict contraction. So, by the contraction mapping principle, $\mathscr{F}$ has a unique fixed point $u$, which is clearly the mild solution to (1.1) that we seek. This completes the proof.

Very often, the functional $F$ is generated by a function $f:(0, T) \times X \rightarrow X$ satisfying the Carathéodory conditions. Specifically, we consider (1.2), where $h \in L^{1}(0, T ; X)$ and $f$ satisfies the following conditions:

(H7) (i) $t \mapsto f(t, x)$ is measurable for every $x \in X$, and $f(\cdot, 0) \in L^{1}(0, T ; X)$,

(ii) there exists $M_{f} \in L^{1}(0, T)$ such that $\|f(t, x)-f(t, y)\| \leq M_{f}(t)\|x-y\|$, for all $x, y \in X$ and almost all $t \in(0, T)$.

We claim that the existence and uniqueness of mild solutions to (1.2) can be obtained as a corollary of Theorem 3.2. Indeed, define $F(u)(t)=h(t)-\int_{0}^{t} a(t-s) f(s, u(s)) d s$, for all $u \in C([0, T] ; X)$ and note that $F$ maps $C([0, T] ; X)$ into $L^{1}(0, T ; X)$. Moreover, $\|F u-F v\|_{L^{1}(0, T ; X)} \leq\left\|a * M_{f}\right\|_{L^{1}(0, T)}\|u-v\|_{C([0, T] ; X)}$, so that (H4) is satisfied with $M_{F}=\left\|a * M_{f}\right\|_{L^{1}(0, T)}$. Consequently, (H6) reads

(H8) $M_{S}\left(M_{g}+\left\|a * M_{f}\right\|_{L^{1}(0, T)}\right)<1$.

An application of Theorem 3.2 yields the following result.

TheOREM 3.3. Assume that (H1)-(H3), (H5), (H7), and (H8) are satisfied. Then, for each $h \in L^{1}(0, T ; X)$, (1.2) has a unique mild solution.

We conclude this section with some comments on (1.2) where $g$ is given by

$$
g(u)=u(T), \quad \forall u \in C([0, T] ; X) .
$$

Obviously $g$, as given by (3.5), satisfies (H5) with $M_{g}=1$. Since always $M_{S} \geq 1$, it is 
132 Semilinear Volterra integrodifferential equations ...

clear that condition (H8) does not hold. To incorporate (3.5) in our theory, we consider that the kernel $a$, and the functions $f$ and $h$ are defined on $[0, \infty)$ (rather than on a fixed interval $[0, T])$. Specifically, we assume

(H9) $a \in L^{1}(0, \infty)$;

(H10) $f:[0, \infty) \times X \rightarrow X$ is continuous and satisfies (for some $M>0$ )

$$
\|f(t, x)-f(t, y)\| \leq M\|x-y\|, \quad \forall x, y \in X \text { and } t \geq 0 ;
$$

(H11) $h \in L_{\text {loc }}^{1}([0, \infty) ; X)$.

We also suppose that the pair $(a, A)$ generates a resolvent $S(t)$, in the sense of Definition 2.2 , on $[0, \infty)$ such that

(H12) $\|S(t)\|_{\mathscr{B}(X)} \leq L e^{-\omega t}$, for all $t \geq 0$,

for some constants $L \geq 1$ and $\omega>0$. For conditions on $a$ and $A$ that guarantee (H12), see [12] and [19, pages 42-44]. (Note, in particular, that (H9) and (H12) are compatible.) We can now prove that the problem

$$
\begin{gathered}
u^{\prime}(t)+\int_{0}^{t} a(t-s)[A u(s)+f(s, u(s))] d s=h(t), \quad 0<t<T, \\
u(0)=u(T)
\end{gathered}
$$

has a unique mild solution, provided that $T$ is large enough.

THEOREM 3.4. Let (H1), (H9)-(H13) be satisfied. If also

(H13) $L \exp \left(-\omega T+L M\|a\|_{L^{1}(0, \infty)}\right)<1$,

then (3.7) has a unique mild solution on $[0, T]$.

Proof. By standard arguments, it follows that for each (fixed) $T>0$ and $x \in X$, the initial-value problem

$$
\begin{gathered}
u^{\prime}(t)+\int_{0}^{t} a(t-s)[A u(s)+f(s, u(s))] d s=h(t), \quad 0<t<T, \\
u(0)=x
\end{gathered}
$$

has a unique mild solution $u_{x}$ on $[0, T]$, with $u_{x}$ given by

$$
u_{x}(t)=S(t) x+S * h(t)-S *\left(a * f\left(\cdot, u_{x}(\cdot)\right)\right)(t) .
$$

On account of (H9), (H10), and (H12), (3.9) yields

$$
\left\|u_{x}(t)-u_{y}(t)\right\| \leq L e^{-\omega t}\|x-y\|+L M \int_{0}^{t} e^{-\omega(t-s)}\|a\|_{L^{1}(0, s)}\left\|u_{x}(s)-u_{y}(s)\right\| d s,
$$

for all $x, y \in X$ and all $t \in[0, T]$. Employing (H9) and a Gronwall type inequality in (3.10) (cf., [15, Lemma 4.2]), we arrive at

$$
\left\|u_{x}(T)-u_{y}(T)\right\| \leq L \exp \left(-\omega T+L M\|a\|_{L^{1}(0, \infty)}\right)\|x-y\|, \quad \forall x, y \in X .
$$


Define $Q_{T}: X \rightarrow X$ by $Q_{T} x=u_{x}(T)$, for all $x \in X$, and observe that (3.11) and (H13) imply that $Q_{T}$ is a contraction on $X$ for a sufficiently large $T$. Therefore, if $T$ is chosen such that (H13) is satisfied, $Q_{T}$ has a unique fixed point $x_{0}$. The corresponding $u_{x_{0}}=u$ is obviously the (unique) mild solution of (3.7) and the proof is complete.

Remark 3.5. It is easy to generalize Theorem 3.4 to the case when (1.2a) is coupled with a nonlocal condition of the form (1.3b). The details are left to the reader.

\section{The case when $A^{-1}$ is compact}

We now investigate (1.1) in the case where the Lipschitz conditions on $F$ and $g$ are dropped. The following assumptions will be used instead.

(H14) $F: C([0, T] ; X) \rightarrow L^{p}(0, T ; X)(p \in(1, \infty])$ is a continuous hereditary map satisfying

$$
\|F v\|_{L^{p}(0, T ; X)} \leq c_{1}\|v\|_{C([0, T] ; X)}+c_{2}, \quad \forall v \in C([0, T] ; X),
$$

where $c_{1}, c_{2}>0$;

(H15) $g: C([0, T] ; X) \rightarrow X$ is a continuous, compact map such that

$$
\|g(u)\| \leq d_{1}\|u\|_{C([0, T] ; X)}+d_{2}, \quad \forall u \in C([0, T] ; X),
$$

where $d_{1}, d_{2}>0$.

Theorem 4.1. Assume that (C1), (C2), (C3)(either (i) or (ii)), (2.5), (H14), and (H15) are satisfied. If, in addition, $1-M_{S}\left(d_{1}+c_{1} T^{1 / q}\right)>0$, then (1.1) has at least one mild solution on $[0, T]$.

Proof. Consider (3.3) and define the solution map $\mathscr{F}$ as in the proof of Theorem 3.2. We appeal to Schaefer's theorem to prove that $\mathscr{F}$ has a fixed point. The continuity of $\mathscr{F}$ is easily verified. We show that $\mathscr{F}$ is a compact map. Let $K_{r}=\{v \in C([0, T] ; X)$ : $\left.\|v\|_{C([0, T] ; X)} \leq r\right\}$ and observe that (cf. (2.3) and (2.4)) $\mathscr{F}_{F} v=S(\cdot) g(v)+\Phi(F(v))$, for all $v \in K_{r}$. We claim that $\mathscr{F}\left(K_{r}\right)$ is precompact in $C([0, T] ; X)$. First, $\{\Phi(F(v)): v \in$ $\left.K_{r}\right\}$ is precompact in $C([0, T] ; X)$ on account of Proposition 2.4 since $\left\{F(v): v \in K_{r}\right\}$ is a bounded subset of $L^{p}(0, T ; X)$ (cf. (H14)). It remains to show that $\{S(\cdot) g(v)$ : $\left.v \in K_{r}\right\}$ is precompact in $C([0, T] ; X)$. To this end, note that by $(\mathrm{H} 15)$ the set $K=$ $\left\{g(v): v \in K_{r}\right\}$ is precompact in $X$. Let $\tilde{K}=S(\cdot) K(\subset C([0, T] ; X))$ and $\epsilon>0$. Since $K$ is precompact in $X$, there exist $\left\{x_{1}, \ldots, x_{n}\right\} \subset K$ such that $K \subset \cup_{i=1}^{n} B\left(x_{i}, \epsilon / M_{S}\right)$. (Here, $B\left(x_{i}, \epsilon / M_{S}\right)$ denotes the ball in $X$ with radius $\epsilon / M_{S}$ centered at $x_{i}$.) Then $\tilde{K} \subset$ $\cup_{i=1}^{n} S(\cdot) B\left(x_{i}, \epsilon / M_{S}\right)$. Set $\tilde{x}_{i}=S(\cdot) x_{i}(\in C([0, T] ; X))$ and $\tilde{B}_{i}=\{y \in C([0, T] ; X)$ : $\left.\left\|y-\tilde{x}_{i}\right\|_{C([0, T] ; X)}<\epsilon\right\}$. If $z \in \tilde{K}$, there exists $\xi \in K$ such that $z=S(\cdot) \xi$. Since $\xi \in K$, there is an $i \in\{1, \ldots, n\}$ such that $\left\|\xi-x_{i}\right\|<\epsilon / M_{S}$. Observe that $\left\|z-\tilde{x}_{i}\right\|_{C([0, T] ; X)}=$ $\left\|S(\cdot) \xi-S(\cdot) x_{i}\right\|_{C([0, T] ; X)} \leq M_{S}\left\|\xi-x_{i}\right\|<\epsilon$. It follows that $\tilde{K} \subset \cup_{i=1}^{n} \tilde{B}_{i}$ and therefore, it is totally bounded. Thus, $\tilde{K}$ is precompact in $C([0, T] ; X)$, as desired.

To apply Schaefer's fixed point theorem, we also need to show that the set $\xi(\mathscr{F})$, as defined in Theorem 2.6 (with $C([0, T] ; X)$ in place of $X)$ is bounded. If $v \in \xi(\mathscr{F})$ and 
134 Semilinear Volterra integrodifferential equations ...

$0 \leq t \leq T$, we have by (3.2), (H14), and (H15)

$$
\begin{aligned}
\lambda\|v(t)\| & \leq\|S(t)\|\|g(v)\|+\int_{0}^{t}\|S(t-s)\|\|(F v)(s)\| d s \\
& \leq M_{S}\left[d_{1}\|v\|_{C([0, T] ; X)}+d_{2}+T^{1 / q}\|F v\|_{L^{p}(0, T ; X)}\right] \\
& \leq M_{S}\left[d_{1}+c_{1} T^{1 / q}\right]\|v\|_{C([0, T] ; X)}+M_{S}\left[d_{2}+c_{2} T^{1 / q}\right],
\end{aligned}
$$

where $M_{S}$ has the same meaning as in (H3). Taking into account that $\lambda \geq 1$ and $M_{S}\left[d_{1}+c_{1} T^{1 / q}\right]<1$ we conclude that $\|v\|_{C([0, T] ; X)} \leq C$. (We hereafter employ $C$ to denote a generic positive constant.) Hence, $\xi(\mathscr{F})$ is bounded and consequently $\mathscr{F}$ has a fixed point $u \in C([0, T] ; X)$. Obviously, $u$ satisfies (3.2) and the proof is complete.

Next, we consider (1.2) under conditions similar to those in Theorem 4.1. Precisely, we replace assumption (H14) by

(H16) $f:[0, T] \times X \rightarrow X$ is a map satisfying the Carathéodory conditions (i.e., $f$ is measurable in $t$ and continuous in $x$ ) and $\|f(t, x)\| \leq c_{1}(t)\|x\|+c_{2}(t)$, for almost all $t \in(0, T)$ and all $x \in X$, where $c_{1}, c_{2} \in L^{1}(0, T)$.

Theorem 4.2. Assume that (C1), (C2), (C3)(either (i) or (ii)), (2.6), and (H16) are satisfied. If, in addition, $1-M_{S}\left(d_{1}+\left\|a * c_{1}\right\|_{L^{1}(0, T)}\right)>0$ and $h \in L^{1}(0, T ; X)$, then (1.2) has at least one mild solution on $[0, T]$.

Proof. Let $v \in C([0, T] ; X)$ and consider the problem

$$
\begin{gathered}
u^{\prime}(t)+\int_{0}^{t} a(t-s)[A u(s)+f(s, v(s))] d s=h(t), \quad 0<t<T, \\
u(0)=g(v) .
\end{gathered}
$$

Define $\mathscr{F}: C([0, T] ; X) \rightarrow C([0, T] ; X)$ by $\mathscr{F} v=u_{v}$, where $u_{v}$ is the mild solution of (4.4). Invoking (2.3) and (2.4), we can write, for $v \in C([0, T] ; X)$,

$$
(\mathscr{F} v)(t)=S(t) g(v)+S * h(t)-\Phi(a * f(\cdot, v(\cdot)))(t) .
$$

Again, we use Schaefer's theorem to establish that $\mathscr{F}$ has at least one fixed point. The continuity of $\mathscr{F}$ follows easily. Let $r>0$ and define the set $K_{r}$ as in the proof of Theorem 4.1. We show that $\mathscr{F}\left(K_{r}\right)$ is precompact in $C([0, T] ; X)$. Since $\{S(\cdot) g(v)$ : $\left.v \in K_{r}\right\}$ was earlier shown to be precompact in $C([0, T] ; X)$ and $S * h$ is independent of $v$, we only focus on the last term on the right-hand side of (4.5). By (H16), we have $\|f(\cdot, v(\cdot))\|_{L^{1}(0, T ; X)} \leq\left\|c_{1}\right\|_{L^{1}(0, T)} r+\left\|c_{2}\right\|_{L^{1}(0, T)}$, for all $v \in K_{r}$. Hence, Proposition 2.5 implies that $\left\{\Phi(a * f(\cdot, v(\cdot))): v \in K_{r}\right\}$ is precompact in $C([0, T] ; X)$. Thus, $\mathscr{F}$ is a compact map.

Finally, let $\xi(\mathscr{F})=\{v \in C([0, T] ; X): \lambda v=\mathscr{F} v$, for some $\lambda \geq 1\}$ and let $v \in \xi(\mathscr{F})$. We seek a constant $C$, independent of $\lambda$ and $v$, such that $\|v\|_{C([0, T] ; X)} \leq C$, for all 
$v \in \xi(\mathscr{F})$. Owing to (H15) and (H16), we have for $t \in[0, T]$,

$$
\begin{aligned}
\lambda\|v(t)\| \leq & \|S(t) g(v)\|+\int_{0}^{t}\|S(t-s)\|\left[\|h(s)\|+\int_{0}^{s}|a(s-\tau)|\|f(\tau, v(\tau))\| d \tau\right] d s \\
\leq & M_{S}\left(d_{1}\|v\|_{C([0, T] ; X)}+d_{2}\right)+M_{S}\|h\|_{L^{1}(0, T)} \\
& +M_{S}\left(\left\|a * c_{1}\right\|_{L^{1}(0, T)}\|v\|_{C([0, T] ; X)}+\|a\|_{L^{1}(0, T)}\left\|c_{2}\right\|_{L^{1}(0, T)}\right) \\
= & M_{S}\left[\left(d_{1}+\left\|a * c_{1}\right\|_{L^{1}(0, T)}\right)\|v\|_{C([0, T] ; X)}+d_{2}+\|h\|_{L^{1}(0, T)}\right. \\
& \left.\quad+\|a\|_{L^{1}(0, T)}\left\|c_{2}\right\|_{L^{1}(0, T)}\right] .
\end{aligned}
$$

Recalling that $\lambda \geq 1$ and $M_{S}\left(d_{1}+\left\|a * c_{1}\right\|_{L^{1}(0, T)}\right)<1$, (4.6) yields the desired bound for $\|v\|_{C([0, T] ; X)}$. Therefore, we conclude by Theorem 2.6 that $\mathscr{F}$ has a fixed point, which is a mild solution to (1.2). This completes the proof.

We can further weaken the assumption on $f$ by replacing $(\mathrm{H} 16)$ with

(H17) $f:[0, T] \times X \rightarrow X$ satisfies the Carathéodory conditions, as well as

(i) $\forall k \in \mathbb{N}, \exists g_{k} \in L^{1}\left(0, T ; \mathbb{R}^{+}\right)$such that for a.a. $t \in(0, T)$,

$\sup _{\|x\| \leq k}\|f(t, x)\| \leq g_{k}(t)$

(ii) $\underline{\lim } k^{-1} \int_{0}^{T}\left(|a| * g_{k}\right)(s) d s=\beta<\infty$.

Theorem 4.3. Assume that (C1), (C2), (C3)(either (i) or (ii)), (2.6), (H15), and (H17) are satisfied. If, in addition, $M_{S}\left(d_{1}+\beta\right)<1$, then for every $h \in L^{1}(0, T ; X)$, (1.2) has at least one mild solution on $[0, T]$.

Proof. Consider (4.4) and define the solution map $\mathscr{F}$ by (4.5). We now use Schauder's fixed point theorem to show that $\mathscr{F}$ has a fixed point. First, we remark that $\mathscr{F}_{F}$ is continuous and compact in $C([0, T] ; X)$; see the proof of Theorem 4.2. It remains to prove that $\mathscr{F}$ maps some ball in $C([0, T] ; X)$ into itself. For each $n \in \mathbb{N}$, let $B_{n}=\left\{x \in C([0, T] ; X):\|x\|_{C([0, T] ; X)} \leq n\right\}$. We show that there is an $n \in \mathbb{N}$ such that $\mathscr{F} B_{n} \subseteq B_{n}$. Suppose, by contradiction, that for each $k \in \mathbb{N}$, there exists $u_{k} \in B_{k}$ such that $\mathscr{F} u_{k} \notin B_{k}$. Then

$$
1 \leq \underline{\lim }_{k \rightarrow \infty} k^{-1}\left\|\mathscr{F} u_{k}\right\|_{C([0, T] ; X)} .
$$

Observe that

$$
\left\|\mathscr{F} u_{k}(t)\right\| \leq\|S(t)\|\left\|g\left(u_{k}\right)\right\|+\|S\| *\left(\|h\|+|a| *\left\|f\left(\cdot, u_{k}(\cdot)\right)\right\|\right)(t), \quad \forall t \in[0, T] .
$$

Since $u_{k} \in B_{k}$, there exists $g_{k} \in L^{1}(0, T)$ such that $\| f\left(\tau, u_{k}(\tau) \| \leq g_{k}(\tau)\right)$ for a.a. $\tau \in(0, T)$ (cf. (H17)(i)). Then, (4.8) leads to

$$
\left\|\mathscr{F} u_{k}(t)\right\| \leq M_{S}\left(\left\|g\left(u_{k}\right)\right\|+\|h\|_{L^{1}(0, T ; X)}+\left\|a * g_{k}\right\|_{L^{1}(0, T)}\right), \quad \forall t \in[0, T] .
$$


136 Semilinear Volterra integrodifferential equations ...

Using (H15) and (H17)(ii) in (4.9), we arrive at $\underline{\lim }_{k \rightarrow \infty} k^{-1} \| \mathscr{F}_{k} u_{C([0, T] ; X)} \leq$ $M_{S}\left(d_{1}+\beta\right)<1$. This contradicts (4.7), so there must exist an $n_{0} \in \mathbb{N}$ such that $\mathscr{F} B_{n_{0}} \subseteq B_{n_{0}}$. Thus, Schauder's fixed point theorem is applicable and the proof is complete.

\section{Examples}

Let $\Omega$ be a bounded domain in $\mathbb{R}^{N}(N \geq 1)$ with a smooth boundary $\Gamma$. Consider the initial-boundary value problem

$$
\begin{gathered}
u_{t}(t, x)+\int_{0}^{t} a(t-s)\left[-\Delta_{x} u(s, x)+\tilde{f}(s, u(s, x))\right] d s=h(t, x), \quad \text { a.e. on }(0, T) \times \Omega, \\
u(t, x)=0, \quad \text { a.e. on }(0, T) \times \Gamma, \\
\left.u(0, x)=\sum_{i=1}^{p} c_{i} u\left(t_{i}, x\right), \quad \text { a.e. on } \Omega .1\right)
\end{gathered}
$$

Here, $0<t_{1}<t_{2}<\cdots<t_{p} \leq T$ are given, $c_{i}>0(i=1, \ldots, p), h:[0, T] \times \Omega \rightarrow \mathbb{R}$, $a:(0, T) \rightarrow \mathbb{R}$, and $\tilde{f}:[0, T] \times \Omega \rightarrow \mathbb{R}$.

THEOREM 5.1. Let (C1) be satisfied. In addition, assume:

(H18) $h \in L^{1}\left(0, T ; L^{2}(\Omega)\right)$;

(H19) $\tilde{f}$ satisfies the Carathéodory conditions, $\tilde{f}(t, 0) \in L^{1}(0, T)$, and there exists $M_{\tilde{f}} \in L^{1}(0, T)$ such that $|\tilde{f}(t, x)-\tilde{f}(t, y)| \leq M_{\tilde{f}}(t)|x-y|, \forall x, y \in \Omega$, a.e. on $(0, T)$;

(H20) $\sum_{i=1}^{p} c_{i}+\left\|M_{\tilde{f}} * a\right\|_{L^{1}(0, T)}<1$.

Then the problem (5.1),(5.2), and (5.3) has a unique mild solution $u \in C\left([0, T] ; L^{2}(\Omega)\right)$.

Proof. Let $X=L^{2}(\Omega)$ and define $A: D(A) \subseteq X \rightarrow X$ by

$$
A u=-\Delta u, \quad D(A)=H^{2}(\Omega) \cap H_{0}^{1}(\Omega) .
$$

It is well known that $A$ is a positive definite, self-adjoint operator in $X$. Moreover, by (C1) and [20, page 38], condition (H3) is satisfied with $M_{S}=1$. Next, $\tilde{f}$ generates a function $f:[0, T] \times X \rightarrow X$ by the formula $f(t, v)(x)=\tilde{f}(t, v(x))$, a.e. on $(0, T) \times \Omega$, for all $v \in X$. Clearly, by (H19), $f$ satisfies (H7). Finally, define $g: C([0, T] ; X) \rightarrow X$ by $g(u)=\sum_{i=1}^{p} c_{i} u\left(t_{i}, x\right)$ and observe that $\|g(u)-g(v)\| \leq M_{g}\|u-v\|_{C([0, T] ; X)}$, with $M_{g}=\sum_{i=1}^{p} c_{i}$ (that is, (H5) holds). Assumption (H8) is also satisfied because of (H20).

We can now rewrite (5.1), (5.2), and (5.3) in the form (1.2) in $X$, and apply Theorem 3.3 to conclude that the problem (5.1), (5.2), and (5.3) has a unique mild solution $u \in C\left([0, T] ; L^{2}(\Omega)\right)$.

Next, consider (5.1) (with $\tilde{f}(s, u(s, x))$ replaced by $\tilde{f}(s, x, u(s, x)))$ and (5.2), together with the new boundary condition

$$
u(0, x)=\int_{\Omega} \int_{0}^{T} \tilde{g}(t, x, z, u(t, z)) d t d z \quad \text { a.e. on } \Omega,
$$


where $\tilde{f}:[0, T] \times \Omega \times \mathbb{R} \rightarrow \mathbb{R}$ and $\tilde{g}:[0, T] \times \Omega \times \Omega \times \mathbb{R} \rightarrow \mathbb{R}$ are given.

The following conditions will be imposed:

(H21) $\tilde{f}:[0, T] \times \Omega \times \mathbb{R} \rightarrow \mathbb{R}$ is a function satisfying the Carathéodory conditions (that is, $\tilde{f}$ is measurable in $(t, x)$ and continuous in the third variable), as well as the growth condition $|\tilde{f}(t, x, u)| \leq m_{1}(t)|u|+m_{2}(t, x)$, for a.a. $(t, x) \in$ $(0, T) \times \mathbb{R}$ and all $u \in \mathbb{R}$, where $m_{1} \in L^{1}\left(0, T ; \mathbb{R}^{+}\right)$and $m_{2} \in L^{1}\left(0, T ; L^{2}(\Omega)\right) ;$

(H22) (i) for almost every $(t, x, z) \in(0, T) \times \Omega \times \Omega, \tilde{g}$ is a continuous function of $r$,

(ii) for each fixed $r \in \mathbb{R}, \tilde{g}$ is a measurable function of $(t, x, z)$,

(iii) $|\tilde{g}(t, x, z, r)-\tilde{g}(t, \bar{x}, z, r)| \leq \gamma_{k}(t, x, \bar{x}, r)$ for $(t, x, z, r),(t, \bar{x}, z, r)$ in $[0, T] \times \Omega \times \Omega \times \mathbb{R}$ with $|r| \leq k$, where $\gamma_{k} \in L^{1}\left((0, T) \times \Omega \times \Omega \times \mathbb{R} ; \mathbb{R}^{+}\right)$ satisfies $\lim _{x \rightarrow \bar{x}} \int_{\Omega} \int_{0}^{T} \gamma_{k}(t, x, \bar{x}, r) d t d z=0$ uniformly in $\bar{x} \in \Omega$,

(iv) $|\tilde{g}(t, x, z, r)| \leq(\delta / T m(\Omega))|r|+\sigma(t, x, z), \forall r \in \mathbb{R}$, a.e. on $(0, T) \times \Omega \times \Omega$, where $\sigma \in L^{2}\left((0, T) \times \Omega \times \Omega ; \mathbb{R}^{+}\right), \delta>0$, and $m(\Omega)$ denotes the Lebesgue measure of $\Omega$;

(H23) $\beta+\delta<1$, where $\beta=\left\|a * m_{1}\right\|_{L^{1}(0, T)}$.

THEOREM 5.2. Let (H18), (H21), (H22), and (H23) be satisfied. If also (C1) holds, and $-a^{\prime}$ is convex, then the problem (5.1) (with $\tilde{f}(s, x, u)$ in place of $\left.f(s, u)\right),(5.2),(5.5)$ has at least one mild solution $u \in C\left([0, T] ; L^{2}(\Omega)\right)$.

Proof. Let $X=L^{2}(\Omega)$ and define $A$ as in (5.4). Define $f:[0, T] \times X \rightarrow X$ and $g: C([0, T] ; X) \rightarrow X$ by

$$
\begin{gathered}
f(t, u)(x)=\tilde{f}(t, x, u(x)), \quad \forall u \in X, \text { a.e. on } \Omega, \\
g(u)(x)=\int_{\Omega} \int_{0}^{T} \tilde{g}(t, x, z, u(t, z)) d t d z, \quad \text { a.e. on } \Omega,
\end{gathered}
$$

respectively, and remark that our problem can be rewritten in the abstract form (1.2) in $X$.

We show that Theorem 4.3 is applicable. First, note that from (5.6) and (H21), it follows that $f$ is a well-defined mapping from $[0, T] \times X$ into $X$ which satisfies the Carathéodory conditions. Let $k \in \mathbb{N}$ be fixed and $u \in X$ be such that $\|u\| \leq k$. Then, (H21) implies that $\|f(t, u)\| \leq m_{1}(t) k+\left\|m_{2}(t, \cdot)\right\|=g_{k}(t)$, with $g_{k} \in L^{1}\left(0, T ; \mathbb{R}^{+}\right)$. Moreover,

$$
\begin{aligned}
\varliminf_{k \rightarrow \infty} k^{-1} \int_{0}^{T}\left(|a| * g_{k}\right)(s) d s & =\varliminf_{k \rightarrow \infty} k^{-1} \int_{0}^{T}\left(|a| *\left(m_{1}(s) k+\left\|m_{2}(s, \cdot)\right\|\right)\right)(s) d s \\
& \leq \varliminf_{k \rightarrow \infty}^{\lim } k^{-1}\left[k\left\|a * m_{1}\right\|_{L^{1}(0, T)}+\|a\|_{L^{1}(0, T)}\left\|m_{2}\right\|_{L^{1}(0, T ; X)}\right] \\
& =\left\|a * m_{1}\right\|_{L^{1}(0, T)}=\beta<\infty,
\end{aligned}
$$

so that (H17) holds. Next, on account of (H22), it follows from Theorem 4.2 in [16, page $175]$ that $g$ is a well-defined, continuous compact map. In addition, for $u \in C([0, T] ; X)$ 
we have (cf. (5.7) and (H22)(iv))

$$
\begin{aligned}
|g(u)(x)| & \leq \frac{\delta}{T m(\Omega)} \int_{\Omega} \int_{0}^{T}|u(t, z)| d t d z+\int_{\Omega} \int_{0}^{T} \sigma(t, x, z) d t d z \\
& \leq \frac{\delta}{T \sqrt{m(\Omega)}} \int_{0}^{T}\left[\int_{\Omega}|u(t, z)|^{2} d z\right]^{1 / 2} d t+\int_{\Omega} \int_{0}^{T} \sigma(t, x, z) d t d z \\
& \leq \frac{\delta}{\sqrt{m(\Omega)}}\|u\|_{C\left([0, T] ; L^{2}(\Omega)\right)}+\int_{\Omega} \int_{0}^{T} \sigma(t, x, z) d t d z .
\end{aligned}
$$

Since $\sigma \in L^{2}((0, T) \times \Omega \times \Omega)$, we conclude that $g$ satisfies all conditions of (H15). (In particular, $d_{1}=\delta$.)

Finally, note that (C2) and (C3)(i) are fulfilled because of (5.4) and our assumptions on $a$, and that $M_{S}=1$ in this case. Hence, all conditions of Theorem 4.3 are satisfied. As a result, the conclusion of Theorem 5.2 follows readily.

\section{References}

[1] S. Aizicovici and Y. Gao, Functional-differential equations with nonlocal initial conditions, J. Appl. Math. Stochastic Anal. 10 (1997), no. 2, 145-156. MR 98f:34117. Zbl 883.34065.

[2] S. Aizicovici and K. B. Hannsgen, Local existence for abstract semilinear Volterra integrodifferential equations, J. Integral Equations Appl. 5 (1993), no. 3, 299-313. MR 94j:45005. Zbl 793.45011.

[3] S. Aizicovici and M. McKibben, Existence results for a class of abstract nonlocal Cauchy problems, Nonlinear Anal. 39 (2000), no. 5, Ser. A: Theory Methods, 649-668. MR 2000j:34089. Zbl 991.14562.

[4] K. Balachandran and M. Chandrasekaran, Existence of solutions of a delay differential equation with nonlocal condition, Indian J. Pure Appl. Math. 27 (1996), no. 5, 443-449. MR 96m:34126. Zbl 854.34065.

[5] K. Balachandran and S. Ilamaran, Existence and uniqueness of mild and strong solutions of a semilinear evolution equation with nonlocal conditions, Indian J. Pure Appl. Math. 25 (1994), no. 4, 411-418. MR 95c:34103. Zbl 808.47047.

[6] L. Byszewski, Theorems about the existence and uniqueness of solutions of a semilinear evolution nonlocal Cauchy problem, J. Math. Anal. Appl. 162 (1991), no. 2, 494-505. MR 92m:35005. Zbl 748.34040.

[7] _ Zagadnienia $r$ żniczkowe i funkcjonalno-r żniczkowe $z$ warunkami nielokalnymi [Differential and Functional-Differential Problems with Nonlocal Conditions], Podstawowe Nauki Techniczne. Monografia [Basic Technical Sciences. Monograph], vol. 184, Politechnika Krakowska, Kraków, 1995 (Polish). MR 96e:34139.

[8] L. Byszewski and H. Akca, Existence of solutions of a semilinear functional-differential evolution nonlocal problem, Nonlinear Anal. 34 (1998), no. 1, 65-72. MR 99c:34180. Zbl 934.34068.

[9] L. Byszewski and V. Lakshmikantham, Theorem about the existence and uniqueness of a solution of a nonlocal abstract Cauchy problem in a Banach space, Appl. Anal. 40 (1991), no. 1, 11-19. MR 92h:34121. Zbl 724.34071.

[10] R. W. Carr and K. B. Hannsgen, A nonhomogeneous integro-differential equation in Hilbert space, SIAM J. Math. Anal. 10 (1979), no. 5, 961-984. MR 81b:45014. Zbl 411.45013.

[11] Resolvent formulas for a Volterra equation in Hilbert space, SIAM J. Math. Anal. 13 (1982), no. 3, 459-483. MR 83e:45002. Zbl 501.45015. 
[12] G. Da Prato and M. Iannelli, Linear integro-differential equations in Banach spaces, Rend. Sem. Mat. Univ. Padova 62 (1980), 207-219. MR 82c:45021. Zbl 451.45014.

[13] J. P. Dauer and K. Balachandran, Existence of solutions for an integrodifferential equation with nonlocal condition in Banach spaces, Libertas Math. 16 (1996), 133-143. MR 97g:45005. Zbl 862.45016.

[14] K. B. Hannsgen and R. L. Wheeler, Behavior of the solution of a Volterra equation as a parameter tends to infinity, J. Integral Equations 7 (1984), no. 3, 229-237. MR 86b:45004. Zbl 552.45010.

[15] Y. P. Lin and J. H. Liu, Semilinear integrodifferential equations with nonlocal Cauchy problem, Nonlinear Anal. 26 (1996), no. 5, 1023-1033. MR 96j:34112. Zbl 916.45014.

[16] R. H. Martin, Jr., Nonlinear Operators and Differential Equations in Banach Spaces, Pure and Applied Mathematics, Wiley-Interscience [John Wiley \& Sons], New York, London, Sydney, 1976. MR 58\#11753. Zbl 333.47023.

[17] S. K. Ntouyas and P. Ch. Tsamatos, Global existence for semilinear evolution equations with nonlocal conditions, J. Math. Anal. Appl. 210 (1997), no. 2, 679-687. MR 98e:34116. Zbl 884.34069.

[18] Global existence for semilinear evolution integrodifferential equations with delay and nonlocal conditions, Appl. Anal. 64 (1997), no. 1-2, 99-105. MR 98e:45008. Zbl 874.35126.

[19] J. Prüss, Positivity and regularity of hyperbolic Volterra equations in Banach spaces, Math. Ann. 279 (1987), no. 2, 317-344. MR 89h:45004. Zbl 608.45007.

[20] Evolutionary Integral Equations and Applications, Monographs in Mathematics, vol. 87, Birkhäuser Verlag, Basel, 1993. MR 94h:45010. Zbl 784.45006.

[21] H. Schaefer, Über die methode der a priori-Schranken, Math. Ann. 129 (1955), 415-416 (German). MR 17,175e. Zbl 064.35703.

Sergiu Aizicovici: Department of Mathematics, Ohio University, Athens, Ohio 45701, USA

E-mail address: aizicovi@bing.math.ohiou.edu

Mark McKibben: Department of Mathematics and Computer Science, Goucher ColLEgE, Towson, MARYLAND 21204, USA

E-mail address: mmckibben@goucher.edu 


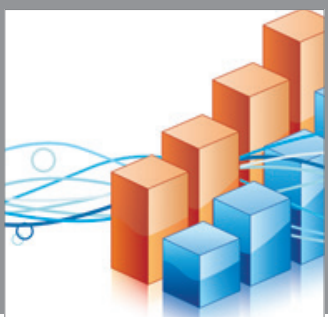

Advances in

Operations Research

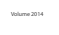

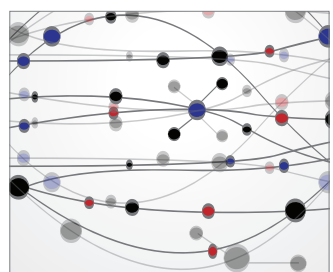

\section{The Scientific} World Journal
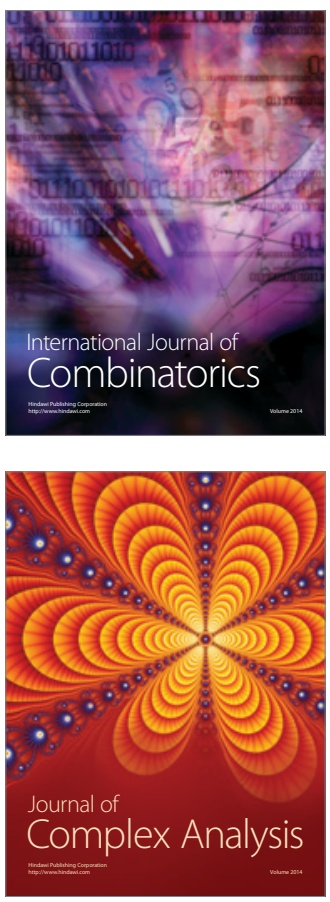

International Journal of

Mathematics and

Mathematical

Sciences
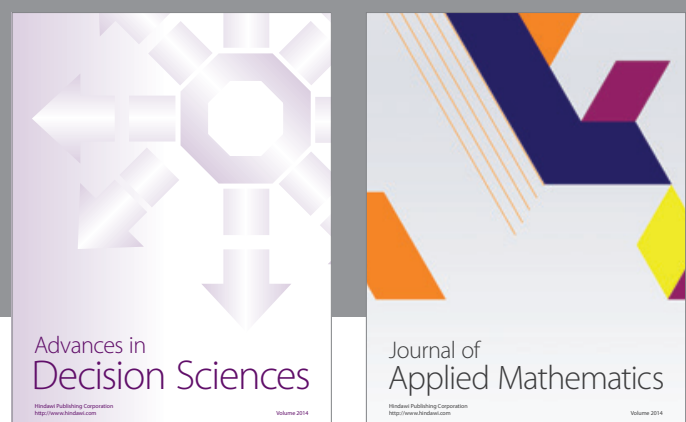

Journal of

Applied Mathematics
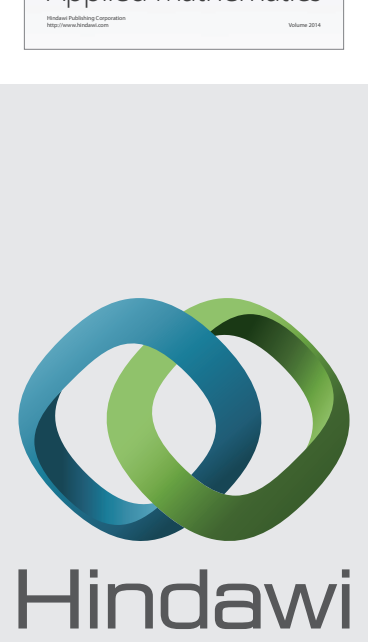

Submit your manuscripts at http://www.hindawi.com
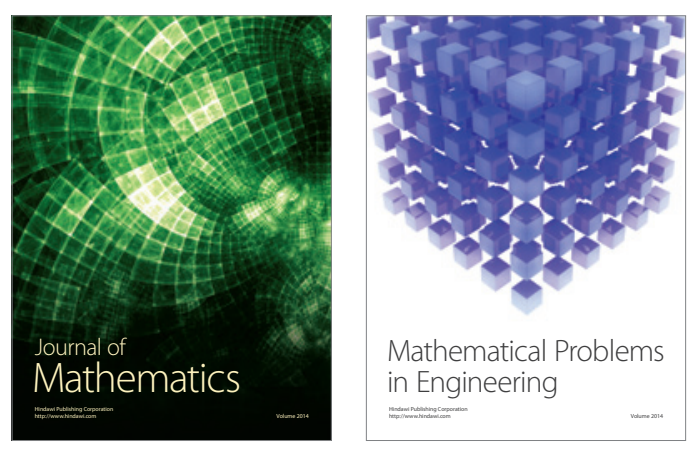

Mathematical Problems in Engineering
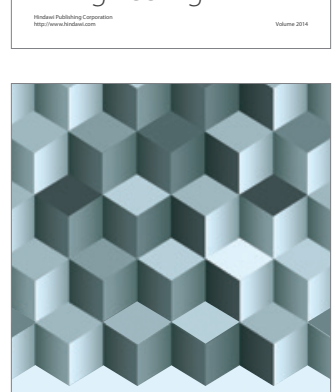

Journal of

Function Spaces
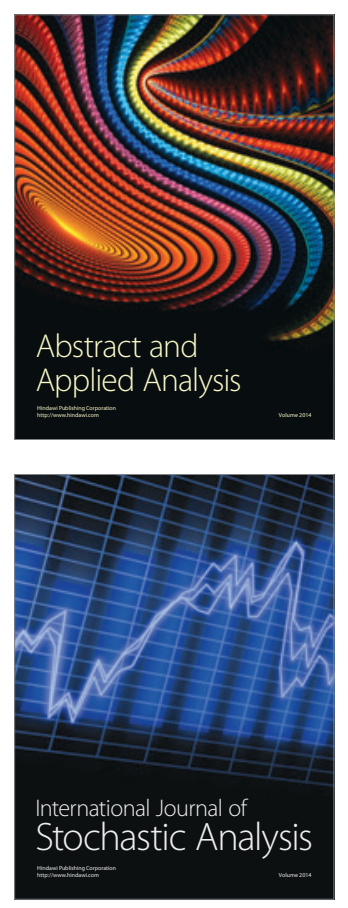

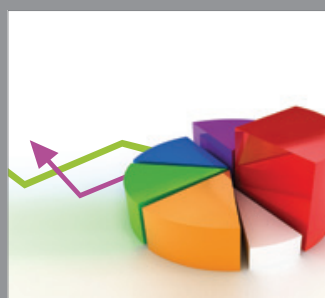

ournal of

Probability and Statistics

Promensencen
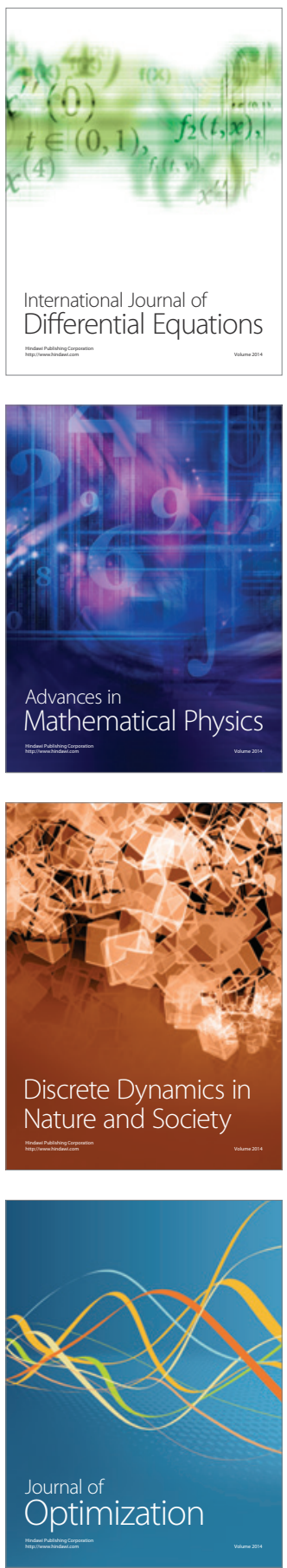